\title{
CONVERGENCE OF OPTION REWARDS FOR MULTIVARIATE PRICE PROCESSES
}

UDC 519.21

\author{
D. S. SILVESTROV AND R. LUNDGREN
}

\begin{abstract}
American type options with general payoff functions possessing polynomial rate of growth are considered for multivariate Markov price processes. Convergence results for optimal reward functionals of American type options for perturbed multivariate Markov processes are presented. These results are applied to approximation tree type algorithms for American type options for exponential multivariate Brownian price processes and mean-reverse price processes used to model stochastic dynamics of energy prices.
\end{abstract}

\section{INTRODUCTION}

The paper present results about convergence of reward functionals for American type options in a multi-asset setting.

Optimal stopping problems for American type options have also been studied in Kim [14], Jacka [9], Peskir and Shiryaev [24], for models with stochastic volatility by Zhang and Lim [35], for American barrier options by Gau, Huang and Subrahmanyam [7, and for generalized American knock out option by Lundgren [18, [19]. Convergence of American option rewards have been studied by Amin and Khanna [1], Schwartz [26], Silvestrov, Galochkin and Sibirtsev [28, Kukush and Silvestrov [15], 16, 17], Jönsson [10], 11], Prigent [25], Jönsson, Kukush and Silvestrov [12], 13], Dupuis and Wang [5], Silvestrov, Jönsson and Stenberg 29], 31, 32, Stenberg [34, Coquet and Toldo [2, Cortazar Gravet and Urzua [3], Lundgren, Silvestrov and Kukush [22], and Lundgren and Silvestrov [20, 21. For recent results concerning optimal stopping we refer to the works of Dayanik and Karatzas [4, Henderson and Hobson [8], and Ekström, Lindberg, Tysk, and Wanntorp [6].

This paper contains 6 sections. In Section 2, we introduce the model of multivariate price processes and American type options with general payoff functions. In Section 3, we present our main results about skeleton type approximations of reward functions and convergence of reward functionals for discrete and continuous time multivariate Markov price processes. We refer to Lundgren and Silvestrov [20] for the proofs, as well as to Silvestrov, Jönsson, and Stenberg [29], 31, 32, where similar results for simpler one-dimensional Markov price processes can be found. In Sections 4, we illustrate our

2000 Mathematics Subject Classification. Primary 60J05, 60H10; Secondary 91B28, 91B70.

Key words and phrases. Reward, convergence, optimal stopping, American option, skeleton approximation, Markov type price process, exponential multivariate Brownian price process, mean-reverse price process.

The paper is based on the talk presented at the International Conference "Modern Stochastics: Theory and Applications II" held on September 7-11, 2010 at Kyiv National Taras Shevchenko University and dedicated to three anniversaries of prominent Ukrainian scientists: AnatoliI SkoroKhod, Volodymyr Korolyuk and Igor Kovalenko. 
general convergence results by applying them to multivariate exponential price processes with independent increments. In Sections 5 and 6, we apply our convergence results to approximation tree type models for American type options for exponential multivariate Brownian price processes and mean-reverse diffusion price processes. Further applications of results presented in this paper relate to the approximation tree type algorithms for the model of optimal reselling of European type options. We refer to the recent papers Lundgren, Silvestrov and Kukush [22] and Lundgren and Silvestrov [21], where one can find the corresponding results.

\section{ReWARD FunCtionals For multivariate Markov PRICE PROCESSES}

For every $\varepsilon \geq 0$, let $\vec{Y}^{(\varepsilon)}(t)=\left(Y_{1}^{(\varepsilon)}(t), \ldots, Y_{k}^{(\varepsilon)}(t)\right), t \geq 0$, be a càdlàg Markov process with the phase space $\mathbb{R}^{k}$ and transition probabilities $P^{(\varepsilon)}(t, \vec{y}, t+s, A)$ and a constant initial state $\vec{Y}^{(\varepsilon)}(0)=\vec{y}_{0} \in \mathbb{R}^{k}$. We interpret $\vec{Y}^{(\varepsilon)}(t)$ as a vector log-price process. Now, we define a vector price process $\vec{S}^{(\varepsilon)}(t)=\left(S_{1}^{(\varepsilon)}(t), \ldots, S_{k}^{(\varepsilon)}(t)\right), t \geq 0$, with the phase space $\mathbb{R}_{+}^{k}=\mathbb{R}_{+} \times \cdots \times \mathbb{R}_{+}$, where $\mathbb{R}_{+}=(0, \infty)$. Let us use the notation $e^{\vec{y}}=\left(e^{y_{1}}, \ldots, e^{y_{k}}\right), \vec{y}=\left(y_{1}, \ldots, y_{k}\right) \in \mathbb{R}^{k}$. The price process $\vec{S}^{(\varepsilon)}(t)$ and the log-price process $\vec{Y}^{(\varepsilon)}(t)$ are connected by the relation $\vec{S}^{(\varepsilon)}(t)=e^{\vec{Y}^{(\varepsilon)}(t)}, t \geq 0$. Due to the oneto-one mapping and continuity property of exponential functions, $\overrightarrow{\vec{S}}(\varepsilon)(t)$ is also a càdlàg Markov process.

Let $g(t, \vec{s}),(t, \vec{s}) \in \mathbb{R}_{+} \times \mathbb{R}_{+}^{k}$ be a payoff function. We assume that $g(t, \vec{s})$ is a real valued Borel measurable function. Note that we do not assume payoff functions to be non-negative. The first condition assumes the absolute continuity of payoff functions and imposes power type upper bounds on their partial derivatives:

$\mathbf{A}_{1}$ : (a) Function $g(t, \vec{s})$ is absolutely continuous in $t$ with respect to the Lebesgue measure on $[0, T]$ for every fixed $\vec{s} \in \mathbb{R}_{+}^{k}$ and in $\vec{s}$ with respect to the Lebesgue measure on $\mathbb{R}_{+}^{k}$ for every fixed $t \in[0, T]$; (b) for every $\vec{s} \in \mathbb{R}_{+}^{k}$, the partial derivative

$$
\left|\frac{\partial g(t, \vec{s})}{\partial t}\right| \leq R_{1}+R_{2} \sum_{j=1}^{k} s_{j}^{\gamma_{0}}
$$

for almost all $t \in[0, T]$ with respect to the Lebesgue measure on $[0, T]$, where $0 \leq R_{1}, R_{2}<\infty$ and $\gamma_{0} \geq 0$; (c) for every $t \in[0, T]$, the partial derivative

$$
\left|\frac{\partial g(t, \vec{s})}{\partial s_{m}}\right| \leq R_{3}+R_{4} \sum_{j=1}^{k} s_{j}^{\gamma_{m}}
$$

for almost all $\vec{s} \in \mathbb{R}_{+}^{k}$ with respect to the Lebesgue measure on $\mathbb{R}_{+}^{k}$, where $0 \leq R_{3}, R_{4}<\infty$ and $\gamma_{1}, \ldots, \gamma_{k} \geq 0, m=1, \ldots, k$. (d) for every $t \in[0, T]$, the function $g(t, \overrightarrow{0})=\varlimsup_{\vec{s} \rightarrow \overrightarrow{0}} g(t, \vec{s}) \leq R_{5}$, where $0 \leq R_{5}<\infty$.

It is useful to note that condition $\mathbf{A}_{\mathbf{1}}$ implies that the function $g(t, \vec{s})$ is continuous in $(t, \vec{s}) \in[0, T] \times \mathbb{R}_{+}^{k}$.

Let $\mathcal{F}_{t}^{(\varepsilon)}=\sigma\left(\vec{Y}^{(\varepsilon)}(s), s \leq t\right)$ be the natural filtration of $\sigma$-fields associated with the vector $\log$-price process $\vec{Y}^{(\varepsilon)}(t), t \geq 0$. It is useful to note that this filtration coincides with the natural filtration generated by the price process $\vec{S}^{(\varepsilon)}(t), t \geq 0$.

We consider Markov moments $\tau^{(\varepsilon)}$ with respect to the filtration $\mathcal{F}_{t}^{(\varepsilon)}, t \geq 0$. It means that $\tau^{(\varepsilon)}$ is a random variable which takes values in $[0, \infty]$ and with the property $\left\{\omega: \tau^{(\varepsilon)}(\omega) \leq t\right\} \in \mathcal{F}_{t}^{(\varepsilon)}, t \geq 0$. Let $\mathcal{M}_{\max , T}^{(\varepsilon)}$ be the class of all Markov moments $\tau^{(\varepsilon)} \leq T$, where $T>0$, and consider a class of Markov moments $\mathcal{M}_{T}^{(\varepsilon)} \subseteq \mathcal{M}_{\max , T}^{(\varepsilon)}$. The main object 
of our studies is the reward functional, that is, the maximal expected payoff over different classes of Markov moments, $\mathcal{M}_{T}^{(\varepsilon)}$ :

$$
\Phi\left(\mathcal{M}_{T}^{(\varepsilon)}\right)=\sup _{\tau^{(\varepsilon)} \in \mathcal{M}_{T}^{(\varepsilon)}} \mathrm{E} g\left(\tau^{(\varepsilon)}, \vec{S}^{(\varepsilon)}\left(\tau^{(\varepsilon)}\right)\right) .
$$

We use the notation $\mathrm{E}_{\vec{y}, t}$ and $\mathrm{P}_{\vec{y}, t}$ for expectation and probability calculated under the condition that $\vec{Y}^{(\varepsilon)}(t)=\vec{y}$. For $\beta, c, T>0, i=1, \ldots, k$, define the exponential moment modulus of compactness for the càdlàg process $Y_{i}^{(\varepsilon)}(t), t \geq 0$ :

$$
\Delta_{\beta}\left(Y_{i}^{(\varepsilon)}(\cdot), c, T\right)=\sup _{0 \leq t \leq t+u \leq t+c \leq T} \sup _{\vec{y} \in \mathbb{R}^{k}} \mathrm{E}_{\vec{y}, t}\left(\exp \left\{\beta\left|Y_{i}^{(\varepsilon)}(t+u)-Y_{i}^{(\varepsilon)}(t)\right|\right\}-1\right) .
$$

We use the following conditions for exponential moment modulus of compactness for log-price processes:

$\mathbf{C}_{\mathbf{1}}: \lim _{c \rightarrow 0} \varlimsup_{\varepsilon \rightarrow 0} \sum_{i=1}^{k} \Delta_{\beta}\left(Y_{i}^{(\varepsilon)}(\cdot), c, T\right)=0$ for some

$$
\beta>\gamma=\max \left(\gamma_{0}, \gamma_{1}+1, \ldots, \gamma_{k}+1\right),
$$

where $\gamma_{0}$ and $\gamma_{1}, \ldots, \gamma_{k}$ are the parameters introduced in condition $\mathbf{A}_{\mathbf{1}}$.

Condition $\mathbf{C}_{\mathbf{1}}$ implies that for any constant $e^{-\beta}<L_{0}<1$ one can choose $c=c\left(L_{0}\right)>0$ and then $\varepsilon_{0}=\varepsilon_{0}(c)$ such that

$$
\frac{\Delta_{\beta}\left(Y_{i}^{(\varepsilon)}(\cdot), c, T\right)+1}{e^{\beta}} \leq L_{0}
$$

for $\varepsilon \leq \varepsilon_{0}$, and $i=1, \ldots, k$.

The following lemma gives asymptotically uniform upper bounds for moments of maximum of price processes, with respect to the perturbation parameter and guarantee that the reward functionals $\Phi\left(\mathcal{M}_{T}^{(\varepsilon)}\right)$ take finite values for all $\varepsilon$ small enough.

Lemma 1. Let conditions $\mathbf{A}_{\mathbf{1}}$ and $\mathbf{C}_{\mathbf{1}}$, hold. Then there exists a constant $L_{1}<\infty$ such that for every $\varepsilon \leq \varepsilon_{0}$,

$$
\sup _{\tau^{(\varepsilon)} \in \mathcal{M}_{\max , T}^{(\varepsilon)}} \mathrm{E}\left|g\left(\tau^{(\varepsilon)}, \vec{S}^{(\varepsilon)}\left(\tau^{(\varepsilon)}\right)\right)\right| \leq \mathrm{E} \sup _{0 \leq u \leq T}\left|g\left(u, \vec{S}^{(\varepsilon)}(u)\right)\right|^{\beta / \gamma} \leq L_{1} .
$$

Let us now assume the following condition of weak convergence (denoted by the symbol $\Rightarrow$ ) for the transition probabilities:

$\mathbf{B}_{1}$ : There exist measurable sets $\mathbb{Y}_{t} \subseteq \mathbb{R}^{k}, t \in[0, T]$, such that:

(a) $P^{(\varepsilon)}\left(t, \vec{y}^{(\varepsilon)}, t+u, \cdot\right) \Rightarrow P^{(0)}(t, \vec{y}, t+u, \cdot)$ as $\varepsilon \rightarrow 0$, for any $\vec{y}^{(\varepsilon)} \rightarrow \vec{y} \in \mathbb{Y}_{t}$ as $\varepsilon \rightarrow 0$ and $0 \leq t<t+u \leq T$

(b) $P^{(0)}\left(t, \vec{y}, t+u, \mathbb{Y}_{t+u}\right)=1$ for every $\vec{y} \in \mathbb{Y}_{t}$ and $0 \leq t<t+u \leq T$;

(c) $\vec{Y}^{(\varepsilon)}(0)=\vec{y}_{0} \in \mathbb{Y}_{0}$.

The following theorem presents our main convergence result. It gives conditions of convergence for reward functionals $\Phi\left(\mathcal{M}_{\max , T}^{(\varepsilon)}\right)$.

Theorem 1. Let conditions $\mathbf{A}_{\mathbf{1}}, \mathbf{B}_{\mathbf{1}}$, and $\mathbf{C}_{\mathbf{1}}$ hold. Then

$$
\Phi\left(\mathcal{M}_{\max , T}^{(\varepsilon)}\right) \rightarrow \Phi\left(\mathcal{M}_{\max , T}^{(0)}\right) \quad \text { as } \varepsilon \rightarrow 0 .
$$

Let $\Pi=\left\{0=t_{0}<t_{1}<\cdots<t_{N}=T\right\}$ be a partition on the interval $[0, T]$ and $d(\Pi)=\max _{1 \leq i \leq N}\left(t_{i}-t_{i-1}\right)$.

We consider the class $\mathcal{M}_{\Pi, T}^{(\varepsilon)}$ of all Markov moments from $\mathcal{M}_{\max , T}^{(\varepsilon)}$, which only take the values $t_{0}, t_{1}, \ldots, t_{N}$ and such that the event $\left\{\omega: \tau^{(\varepsilon)}(\omega)=t_{j}\right\} \in \sigma\left(\vec{Y}^{(\varepsilon)}\left(t_{0}\right), \ldots, \vec{Y}^{(\varepsilon)}\left(t_{j}\right)\right)$ 
for $j=0, \ldots, N$. By definition, $\mathcal{M}_{\Pi, T}^{(\varepsilon)} \subseteq \mathcal{M}_{\max , T}^{(\varepsilon)}$. This relation implies that, under conditions of Lemma $1,-\infty<\Phi\left(\mathcal{M}_{\Pi, T}^{(\varepsilon)}\right) \leq \Phi\left(\mathcal{M}_{\max , T}^{(\varepsilon)}\right)<\infty$.

The reward functionals $\Phi\left(\mathcal{M}_{\max , T}^{(\varepsilon)}\right)$ and $\Phi\left(\mathcal{M}_{\Pi, T}^{(\varepsilon)}\right)$ correspond to American type options in continuous time and American type options in discrete time, respectively. In the first case, the underlying price process is a continuous time Markov type price process, while in the second case, the corresponding price process is a discrete time Markov type process. The random variables $\vec{Y}^{(\varepsilon)}\left(t_{0}\right), \vec{Y}^{(\varepsilon)}\left(t_{1}\right), \ldots, \vec{Y}^{(\varepsilon)}\left(t_{N}\right)$ are connected in a discrete time inhomogeneous Markov chain with the phase space $\mathbb{R}^{k}$, transition probabilities $P^{(\varepsilon)}\left(t_{n}, \vec{y}, t_{n+1}, A\right)$, and the initial state $\vec{Y}^{(\varepsilon)}\left(t_{0}\right)=\vec{y}_{0}$. Note that we have slightly modified the standard definition of a discrete time Markov chain by counting moments $t_{0}, \ldots, t_{N}$ as the moments of jumps for the Markov chain $\vec{Y}^{(\varepsilon)}\left(t_{n}\right)$ instead of the moments $0, \ldots, N$. This is done in order to synchronize the discrete and continuous time models. Thus, the optimization problem (1) for the class $\mathcal{M}_{\Pi, T}^{(\varepsilon)}$ is really a problem of optimal expected rewards for American type options in discrete time.

The following theorem gives a skeleton approximation (asymptotically uniform with respect to perturbation parameter) for reward functionals $\Phi\left(\mathcal{M}_{\max , T}^{(\varepsilon)}\right)$.

Theorem 2. Let conditions $\mathbf{A}_{\mathbf{1}}$ and $\mathbf{C}_{\mathbf{1}}$ hold. Also let $\varepsilon \leq \varepsilon_{0}$ and $d(\Pi) \leq c$, where $\varepsilon_{0}$ and $c$ are defined above. Then there exist constants $L_{2}, L_{3}<\infty$ such that the following skeleton approximation inequality holds, for every $\varepsilon \leq \varepsilon_{0}$ :

$$
\Phi\left(\mathcal{M}_{\max , T}^{(\varepsilon)}\right)-\Phi\left(\mathcal{M}_{\Pi, T}^{(\varepsilon)}\right) \leq L_{2} d(\Pi)+L_{3} \sum_{i=1}^{k} \Delta_{\beta}\left(Y_{i}^{(\varepsilon)}(\cdot), d(\Pi), T\right)^{(\beta-\gamma) / \beta} .
$$

The following theorem gives conditions of convergence for reward functionals:

$$
\Phi\left(\mathcal{M}_{\Pi, T}^{(\varepsilon)}\right) \text {. }
$$

Theorem 3. Let conditions $\mathbf{A}_{\mathbf{1}}, \mathbf{B}_{\mathbf{1}}$, and $\mathbf{C}_{\mathbf{1}}$ hold. Then, the following asymptotic relation holds for any partition $\Pi=\left\{0=t_{0}<t_{1} \cdots<t_{N}=T\right\}$ on the interval $[0, T]$ such that $d(\Pi) \leq c$, where $c$ is defined above:

$$
\Phi\left(\mathcal{M}_{\Pi, T}^{(\varepsilon)}\right) \rightarrow \Phi\left(\mathcal{M}_{\Pi, T}^{(0)}\right) \quad \text { as } \varepsilon \rightarrow 0 .
$$

Let us now formulate some useful sufficient conditions for an important condition of moment compactness $\mathbf{C}_{\mathbf{1}}$.

Let us introduce the modulus of J-compactness, for $h, c>0, i=1, \ldots, k$ :

$$
\Delta\left(Y_{i}^{(\varepsilon)}(\cdot), h, c, T\right)=\sup _{0 \leq t \leq t+u \leq t+c \leq T} \sup _{\vec{y} \in \mathbb{R}^{k}} \mathrm{P}_{\vec{y}, t}\left\{\left|Y_{i}^{(\varepsilon)}(t+u)-Y_{i}^{(\varepsilon)}(t)\right| \geq h\right\} .
$$

The following condition of J-compactness plays the key role in functional limit theorems for Markov type càdlàg processes:

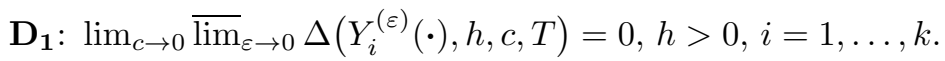

We also introduce the quantity which represents the maximum of moment generating functions for increments of the log-price processes $Y_{i}^{(\varepsilon)}(t), t \geq 0, i=1, \ldots, k$ :

$$
\Xi_{\beta}\left(Y_{i}^{(\varepsilon)}(\cdot), T\right)=\sup _{0 \leq t \leq t+u \leq T} \sup _{\vec{y} \in \mathbb{R}^{k}} \mathrm{E}_{\vec{y}, t} \exp \left\{\beta\left(Y_{i}^{(\varepsilon)}(t+u)-Y_{i}^{(\varepsilon)}(t)\right)\right\}, \quad \beta \in \mathbb{R} .
$$

The following condition formulated in terms of these moment generating functions can be effectively verified in many cases:

$\mathbf{C}_{\mathbf{2}}: \varlimsup_{\lim _{\varepsilon \rightarrow 0}} \Xi_{ \pm \beta^{\prime}}\left(\vec{Y}_{i}^{(\varepsilon)}(\cdot), T\right)<\infty, i=1, \ldots, k$, for some $\beta^{\prime}>\gamma$, where $\gamma$ is the parameter introduced in condition $\mathbf{A}_{\mathbf{1}}$. 
Lemma 2. Conditions $\mathbf{D}_{\mathbf{1}}$ and $\mathbf{C}_{\mathbf{2}}$ imply that condition $\mathbf{C}_{\mathbf{1}}$ holds for any $\beta \in\left(\gamma, \beta^{\prime}\right)$.

\section{EXPONENTIAL PRICE PROCESSES WITH INDEPENDENT INCREMENTS}

In order to illustrate the results given in Theorems 1-3, let us consider the model where the log-price process $\vec{Y}^{(\varepsilon)}(t), t \geq 0$, is a càdlàg process with independent increments, with a constant initial state $\vec{Y}^{(\varepsilon)}(0)=\vec{y}_{0} \in \mathbb{R}^{k}$. The process $\vec{Y}^{(\varepsilon)}(t)$ is a càdlàg Markov process with transition probabilities which are connected with the distributions of increments for this process $P^{(\varepsilon)}(t, t+u, A)$ by the following relation:

$$
\begin{aligned}
P^{(\varepsilon)}(t, \vec{y}, t+u, A) & =P^{(\varepsilon)}(t, t+u, A-\vec{y}) \\
& =\mathrm{P}\left\{\vec{y}+\vec{Y}^{(\varepsilon)}(t+u)-\vec{Y}^{(\varepsilon)}(t) \in A\right\} .
\end{aligned}
$$

Let us assume the following standard condition of weak convergence for distributions of increments for log-price processes:

$\mathbf{B}_{\mathbf{2}}: P^{(\varepsilon)}(t, t+u, \cdot) \Rightarrow P^{(0)}(t, t+u, \cdot)$ as $\varepsilon \rightarrow 0,0 \leq t \leq t+u \leq T$.

Representation (5) implies that condition $\mathbf{B}_{\mathbf{1}}$ holds with the sets $\mathbb{Y}_{t}=\mathbb{R}^{k}, t \in[0, T]$, i.e., distributions of increments for the processes $Y_{i}^{(\varepsilon)}(t)$ locally uniformly weakly converge if and only if condition $\mathbf{B}_{\mathbf{2}}$ holds. Also, condition $\mathbf{B}_{\mathbf{1}}$ (c) automatically holds. Thus, in the case of processes with independent increments, condition $\mathbf{B}_{\mathbf{1}}$, with the sets $\mathbb{Y}_{t}=\mathbb{R}^{k}$ pointed above, is, in fact, equivalent to the standard condition of weak convergence for such processes. In this case the J-compactness modulus $\Delta\left(Y_{i}^{(\varepsilon)}(\cdot), h, c, T\right)$ takes the following form:

$$
\Delta^{\prime}\left(Y_{i}^{(\varepsilon)}(\cdot), h, c, T\right)=\sup _{0 \leq t \leq t+u \leq t+c \leq T} \mathrm{P}\left\{\left|Y_{i}^{(\varepsilon)}(t+u)-Y_{i}^{(\varepsilon)}(t)\right| \geq h\right\} .
$$

Thus, condition $\mathbf{C}_{\mathbf{2}}$ is reduced to the standard J-compactness condition for processes with independent increments:

$\mathbf{D}_{\mathbf{2}}: \varlimsup_{\lim _{c \rightarrow 0}} \lim _{\varepsilon \rightarrow 0} \Delta^{\prime}\left(Y_{i}^{(\varepsilon)}(\cdot), h, c, T\right)=0, h>0, i=1, \ldots, k$.

Note that conditions $\mathbf{B}_{\mathbf{2}}$ and $\mathbf{D}_{\mathbf{2}}$ are necessary and sufficient for J-convergence of processes $\vec{Y}^{(\varepsilon)}(t), t \in[0, T]$, to process $\vec{Y}^{(0)}(t), t \in[0, T]$, as $\varepsilon \rightarrow 0$ and stochastic continuity of the limit process.

Also, the quantities $\Xi_{\beta}\left(Y_{i}^{(\varepsilon)}(\cdot), T\right), i=1, \ldots, k$, take a simplified form,

$$
\Xi_{\beta}^{\prime}\left(Y_{i}^{(\varepsilon)}(\cdot), T\right)=\sup _{0 \leq t \leq t+u \leq T} \mathrm{E} \exp \left\{\beta\left(Y_{i}^{(\varepsilon)}(t+u)-Y_{i}^{(\varepsilon)}(t)\right)\right\}, \quad \beta \in \mathbb{R} .
$$

Therefore, condition $\mathbf{C}_{\mathbf{2}}$ takes the following form:

$\mathbf{C}_{\mathbf{3}}: \varlimsup_{\lim _{\varepsilon \rightarrow 0}} \Xi_{ \pm \beta^{\prime}}^{\prime}\left(Y_{i}^{(\varepsilon)}(\cdot), T\right)<\infty, i=1, \ldots, k$, for some $\beta^{\prime}>\gamma$, where $\gamma$ is the parameter in condition $\mathbf{A}_{\mathbf{1}}$.

According to Lemma 2, conditions $\mathbf{C}_{\mathbf{3}}$ and $\mathbf{D}_{\mathbf{2}}$ imply that condition $\mathbf{C}_{\mathbf{1}}$ holds for any $\beta \in\left(\gamma, \beta^{\prime}\right)$.

The following theorem summarize the remarks above.

Theorem 4. Let conditions $\mathbf{A}_{1}, \mathbf{B}_{\mathbf{2}}, \mathbf{D}_{\mathbf{2}}$, and $\mathbf{C}_{\mathbf{3}}$ hold for the log-price price processes with independent increments $\vec{Y}^{(\varepsilon)}(t)$. Then

$$
\Phi\left(\mathcal{M}_{\max , T}^{(\varepsilon)}\right) \rightarrow \Phi\left(\mathcal{M}_{\max , T}^{(0)}\right) \quad \text { as } \varepsilon \rightarrow 0 .
$$

Note that the asymptotic relation (6) takes place for any initial state $\vec{y}_{0} \in \mathbb{R}^{k}$.

It is worth noting that conditions $\mathbf{A}_{\mathbf{1}}, \mathbf{B}_{\mathbf{2}}, \mathbf{D}_{\mathbf{2}}$, and $\mathbf{C}_{\mathbf{3}}$ imply that the conditions of Theorems 2 and 3 hold for the exponential price processes with independent increments 
$\vec{S}^{(\varepsilon)}(t)$ and, therefore, the skeleton approximation inequality given in Theorem 2 as well as the convergence relation given in Theorem 3 also take place.

\section{Binomial tree approximations for multivariate Brownian motion}

In order to illustrate the results presented above, let us consider the model where $k=2$ and the bivariate geometric Brownian price process $\vec{S}^{(0)}(t)=e^{\vec{Y}^{(0)}(t)}, t \geq 0$, where the log-price process $\vec{Y}^{(0)}(t)=\left(Y_{1}^{(0)}(t), Y_{2}^{(0)}(t)\right), t \geq 0$, is a bivariate Brownian motion with components

$$
Y_{i}^{(0)}(t)=y_{i}^{(0)}+\mu_{i} t+\sigma_{i} W_{i}(t), \quad t \geq 0, i=1,2,
$$

which are correlated, i.e., $\mathrm{E} W_{1}(t) W_{2}(t)=\rho t, t \geq 0$. Note that the log-price process has a constant initial state $\vec{Y}^{(0)}(0)=\vec{y}_{0}=\left(y_{1}^{(0)}, y_{2}^{(0)}\right) \in \mathbb{R}^{2}$.

We approximate the process $\vec{Y}^{(0)}(t), t \geq 0$, with a bivariate binomial sum-process $\vec{Y}^{(\varepsilon)}(t)=\left(Y_{1}^{(\varepsilon)}(t), Y_{2}^{(\varepsilon)}(t)\right), t \geq 0$, with components

$$
Y_{i}^{(\varepsilon)}(t)=y_{i}^{(0)}+\sum_{1 \leq k \leq[t / \varepsilon]} Y_{k, i}^{(\varepsilon)}, \quad t \geq 0, i=1,2 .
$$

Here $\vec{Y}_{n}^{(\varepsilon)}=\left(Y_{n, 1}^{(\varepsilon)}, Y_{n, 2}^{(\varepsilon)}\right), n=1,2, \ldots$, are, for every $\varepsilon>0$, i.i.d. random vectors which have the following structure:

$$
\left(Y_{n, 1}^{(\varepsilon)}, Y_{n, 2}^{(\varepsilon)}\right)=\left\{\begin{aligned}
\left(+u_{1}^{(\varepsilon)},+u_{2}^{(\varepsilon)}\right) & p_{++}^{(\varepsilon)} \\
\left(+u_{1}^{(\varepsilon)},-u_{2}^{(\varepsilon)}\right) & p_{+-}^{(\varepsilon)} \\
\text { with prob. } & \\
\left(-u_{1}^{(\varepsilon)},+u_{2}^{(\varepsilon)}\right) & p_{-+}^{(\varepsilon)} \\
\left.-u_{1}^{(\varepsilon)},-u_{2}^{(\varepsilon)}\right) & p_{--}^{(\varepsilon)} .
\end{aligned}\right.
$$

In order to fit the bivariate binomial sum-processes defined above to the limit bivariate Brownian motion, we should fit expectations, variances, and covariance coefficients for summands $\left(Y_{n, 1}^{(\varepsilon)}, Y_{n, 2}^{(\varepsilon)}\right)$ to the corresponding quantities for the increments of the bivariate Brownian motion

$$
\left(\mu_{1} \varepsilon+\sigma_{1}\left(W_{1}((n+1) \varepsilon)-W_{1}(n \varepsilon)\right), \mu_{2} \varepsilon+\sigma_{2}\left(W_{2}((n+1) \varepsilon)-W_{2}(n \varepsilon)\right)\right) .
$$

The following system of six equations with six unknowns should be solved:

$$
\left\{\begin{array}{l}
\mathrm{E}\left[Y_{1,1}^{(\varepsilon)}\right]=u_{1}^{(\varepsilon)}\left(2\left(p_{++}^{(\varepsilon)}+p_{+-}^{(\varepsilon)}\right)-1\right)=\mu_{1} \varepsilon \\
\operatorname{Var}\left[Y_{1,1}^{(\varepsilon)}\right]=\left(u_{1}^{(\varepsilon)}\right)^{2}-\left(\mu_{1} \varepsilon\right)^{2}=\sigma_{1}^{2} \varepsilon \\
\mathrm{E}\left[Y_{1,2}^{(\varepsilon)}\right]=u_{2}^{(\varepsilon)}\left(2\left(p_{++}^{(\varepsilon)}+p_{-+}^{(\varepsilon)}\right)-1\right)=\mu_{2} \varepsilon \\
\operatorname{Var}\left[Y_{1,2}^{(\varepsilon)}\right]=\left(u_{2}^{(\varepsilon)}\right)^{2}-\left(\mu_{2} \varepsilon\right)^{2}=\sigma_{2}^{2} \varepsilon \\
\operatorname{Cov}\left[Y_{1,1}^{(\varepsilon)}, Y_{1,2}^{(\varepsilon)}\right]=\frac{u_{1}^{(\varepsilon)} u_{2}^{(\varepsilon)}\left(p_{++}^{(\varepsilon)}+p_{--}^{(\varepsilon)}-p_{-+}^{(\varepsilon)}-p_{+-}^{(\varepsilon)}\right)-\mu_{1} \mu_{2} \varepsilon^{2}}{\sigma_{1} \sigma_{2} \varepsilon}=\rho \\
p_{++}^{(\varepsilon)}+p_{-+}^{(\varepsilon)}+p_{-+}^{(\varepsilon)}+p_{--}^{(\varepsilon)}=1 .
\end{array}\right.
$$


This system has, for every $\varepsilon>0$, the following unique solution:

$$
\left\{\begin{array}{l}
u_{1}^{(\varepsilon)}=\sqrt{\varepsilon} \sqrt{\sigma_{1}^{2}+\mu_{1}^{2} \varepsilon} \\
u_{2}^{(\varepsilon)}=\sqrt{\varepsilon} \sqrt{\sigma_{2}^{2}+\mu_{2}^{2} \varepsilon} \\
p_{++}^{(\varepsilon)}=\frac{1}{4}+\frac{1}{4} \sqrt{\varepsilon}\left(\frac{\mu_{1}}{\sqrt{\sigma_{1}^{2}+\mu_{1}^{2} \varepsilon}}+\frac{\mu_{2}}{\sqrt{\sigma_{2}^{2}+\mu_{2}^{2} \varepsilon}}\right)+\frac{1}{4} \frac{\rho \sigma_{1} \sigma_{2}+\mu_{1} \mu_{2} \varepsilon}{\sqrt{\sigma_{1}^{2}+\mu_{1}^{2} \varepsilon} \sqrt{\sigma_{2}^{2}+\mu_{2}^{2} \varepsilon}}, \\
p_{+-}^{(\varepsilon)}=\frac{1}{4}+\frac{1}{4} \sqrt{\varepsilon}\left(\frac{\mu_{1}}{\sqrt{\sigma_{1}^{2}+\mu_{1}^{2} \varepsilon}}-\frac{\mu_{2}}{\sqrt{\sigma_{2}^{2}+\mu_{2}^{2} \varepsilon}}\right)-\frac{1}{4} \frac{\rho \sigma_{1} \sigma_{2}+\mu_{1} \mu_{2} \varepsilon}{\sqrt{\sigma_{1}^{2}+\mu_{1}^{2} \varepsilon} \sqrt{\sigma_{2}^{2}+\mu_{2}^{2} \varepsilon}}, \\
p_{-+}^{(\varepsilon)}=\frac{1}{4}+\frac{1}{4} \sqrt{\varepsilon}\left(-\frac{\mu_{1}}{\sqrt{\sigma_{1}^{2}+\mu_{1}^{2} \varepsilon}}+\frac{\mu_{2}}{\sqrt{\sigma_{2}^{2}+\mu_{2}^{2} \varepsilon}}\right)-\frac{1}{4} \frac{\rho \sigma_{1} \sigma_{2}+\mu_{1} \mu_{2} \varepsilon}{\sqrt{\sigma_{1}^{2}+\mu_{1}^{2} \varepsilon} \sqrt{\sigma_{2}^{2}+\mu_{2}^{2} \varepsilon}}, \\
p_{--}^{(\varepsilon)}=\frac{1}{4}+\frac{1}{4} \sqrt{\varepsilon}\left(-\frac{\mu_{1}}{\sqrt{\sigma_{1}^{2}+\mu_{1}^{2} \varepsilon}}-\frac{\mu_{2}}{\sqrt{\sigma_{2}^{2}+\mu_{2}^{2} \varepsilon}}\right)+\frac{1}{4} \frac{\rho \sigma_{1} \sigma_{2}+\mu_{1} \mu_{2} \varepsilon}{\sqrt{\sigma_{1}^{2}+\mu_{1}^{2} \varepsilon} \sqrt{\sigma_{2}^{2}+\mu_{2}^{2} \varepsilon}} .
\end{array}\right.
$$

It is useful to note that the corresponding parameter have the following asymptotic expansions:

$$
\left\{\begin{array}{l}
u_{1}^{(\varepsilon)}=\sqrt{\varepsilon} \sigma_{1}+o(\varepsilon), \\
u_{2}^{(\varepsilon)}=\sqrt{\varepsilon} \sigma_{2}+o(\varepsilon), \\
p_{++}^{(\varepsilon)}=\frac{1}{4}+\frac{1}{4} \rho+\frac{1}{4}\left(\frac{\mu_{1}}{\sigma_{1}}+\frac{\mu_{2}}{\sigma_{2}}\right) \sqrt{\varepsilon}+\frac{1}{4}\left(\frac{\mu_{1} \mu_{2}}{\sigma_{1} \sigma_{2}}-\frac{\rho \mu_{1}^{2}}{2 \sigma_{1}^{2}}-\frac{\rho \mu_{2}^{2}}{2 \sigma_{2}^{2}}\right) \varepsilon+o(\varepsilon), \\
p_{+-}^{(\varepsilon)}=\frac{1}{4}-\frac{1}{4} \rho+\frac{1}{4}\left(\frac{\mu_{1}}{\sigma_{1}}-\frac{\mu_{2}}{\sigma_{2}}\right) \sqrt{\varepsilon}-\frac{1}{4}\left(\frac{\mu_{1} \mu_{2}}{\sigma_{1} \sigma_{2}}-\frac{\rho \mu_{1}^{2}}{2 \sigma_{1}^{2}}-\frac{\rho \mu_{2}^{2}}{2 \sigma_{2}^{2}}\right) \varepsilon+o(\varepsilon), \\
p_{-+}^{(\varepsilon)}=\frac{1}{4}-\frac{1}{4} \rho-\frac{1}{4}\left(\frac{\mu_{1}}{\sigma_{1}}-\frac{\mu_{2}}{\sigma_{2}}\right) \sqrt{\varepsilon}-\frac{1}{4}\left(\frac{\mu_{1} \mu_{2}}{\sigma_{1} \sigma_{2}}-\frac{\rho \mu_{1}^{2}}{2 \sigma_{1}^{2}}-\frac{\rho \mu_{2}^{2}}{2 \sigma_{2}^{2}}\right) \varepsilon+o(\varepsilon), \\
p_{--}^{(\varepsilon)}=\frac{1}{4}+\frac{1}{4} \rho-\frac{1}{4}\left(\frac{\mu_{1}}{\sigma_{1}}+\frac{\mu_{2}}{\sigma_{2}}\right) \sqrt{\varepsilon}+\frac{1}{4}\left(\frac{\mu_{1} \mu_{2}}{\sigma_{1} \sigma_{2}}-\frac{\rho \mu_{1}^{2}}{2 \sigma_{1}^{2}}-\frac{\rho \mu_{2}^{2}}{2 \sigma_{2}^{2}}\right) \varepsilon+o(\varepsilon) .
\end{array}\right.
$$

Relation (10) guarantees that the quantities $p_{ \pm \pm}^{(\varepsilon)}$ take values in the interval $(0,1)$ for $\varepsilon$ small enough in the most interesting non-degenerate case, where $|\rho|<1$. Note also that the sum of these probabilities is equal to 1 , according to the last equation in (8).

The problem can be reduced, however, to the more simple case where drift coefficients $\mu_{1}=\mu_{2}=0$ and the initial state $\vec{y}_{0}=(0,0)$.

Let us consider processes $\overrightarrow{\tilde{S}}^{(0)}(t)=e^{\overrightarrow{\tilde{Y}}^{(0)}(t)}, t \geq 0$, where the log-price process $\overrightarrow{\tilde{Y}}^{(0)}(t)=$ $\left(\tilde{Y}_{1}^{(0)}(t), \tilde{Y}_{2}^{(0)}(t)\right), t \geq 0$, is a bivariate Brownian motion with components $\tilde{Y}_{i}^{(0)}(t)=$ $\sigma_{i} W_{i}(t), t \geq 0, i=1,2$, which are correlated, i.e., $\mathrm{E} W_{1}(t) W_{2}(t)=\rho t, t \geq 0$. Obviously, the natural filtration $\mathcal{F}_{t}, t \geq 0$, is the same for processes $\vec{S}^{(0)}(t), t \geq 0$ and $\overrightarrow{\widetilde{S}}^{(0)}(t), t \geq 0$.

Let $g(t, \vec{s})=g\left(t,\left(s_{1}, s_{2}\right)\right)$ be payoff functions that satisfy conditions $\mathbf{A}_{\mathbf{1}}$. Let us now consider the transformed payoff functions $\tilde{g}(t, \vec{s})=g\left(t,\left(e^{y_{1}^{(0)}+\mu_{1} t} s_{1}, e^{y_{2}^{(0)}+\mu_{2} t} s_{2}\right)\right)$. These functions also satisfy condition $\mathbf{A}_{\mathbf{1}}$ with some constants $K_{i}, i=1, \ldots, 5$, and parameters $\gamma_{0}=\gamma=\max \left(\gamma_{0}, \gamma_{1}+1, \gamma_{2}+1\right)$ and the same parameters $\gamma_{1}$ and $\gamma_{2}$. It follows from the remarks above that the reward functional

$$
\Phi\left(\mathcal{M}_{T}^{(0)}\right)=\sup _{\tau \in \mathcal{M}_{T}} \mathrm{E} g\left(\tau, \vec{S}^{(0)}(\tau)\right)=\sup _{\tau \in \mathcal{M}_{T}} \mathrm{E} \tilde{g}\left(\tau, \overrightarrow{\tilde{S}}^{(0)}(\tau)\right) .
$$

Now, we can approximate the bivariate Brownian processes $\overrightarrow{\tilde{Y}}^{(0)}(t)$ by the corresponding bivariate sum-processes $\overrightarrow{\tilde{Y}}^{(\varepsilon)}(t)$ as described above. In this case, however, the parameters $\mu_{1}$ and $\mu_{2}$ will take the value 0 in systems of equations (8) and (9). In this case, the solution to these systems will take the following simpler form:

$$
\left\{\begin{array}{l}
u_{1}^{(\varepsilon)}=\sqrt{\varepsilon} \sigma_{1}, \\
u_{2}^{(\varepsilon)}=\sqrt{\varepsilon} \sigma_{2}, \\
p_{++}^{(\varepsilon)}=p_{--}^{(\varepsilon)}=\frac{1}{4}+\frac{1}{4} \rho, \\
p_{+-}^{(\varepsilon)}=p_{-+}^{(\varepsilon)}=\frac{1}{4}-\frac{1}{4} \rho .
\end{array}\right.
$$


The probabilities in (12) take non-negative values for any $|\rho| \leq 1$.

By applying convergence theorems for vector sum-processes with independent increments, given for example in Skorokhod [33], it is easy to prove that the processes $\vec{Y}^{(\varepsilon)}(t)$, $t \in[0, T]$, with parameters given in (9) weakly and, moreover, J-converge to process $\vec{Y}^{(0)}(t), t \in[0, T]$, as $\varepsilon \rightarrow 0$. Also the processes $\overrightarrow{\tilde{Y}}^{(\varepsilon)}(t), t \in[0, T]$, with parameters given in (12) weakly and J-converge to process $\overrightarrow{\tilde{Y}}^{(0)}(t), t \in[0, T]$, as $\varepsilon \rightarrow 0$.

Thus, conditions $\mathbf{B}_{\mathbf{2}}$ and $\mathbf{D}_{\mathbf{2}}$ hold for processes $\vec{Y}(\varepsilon)(t)$ and $\overrightarrow{\tilde{Y}}^{(\varepsilon)}(t)$.

Also, the moment generation functions $\operatorname{E} \exp \left\{\beta\left(Y_{i}^{(\varepsilon)}(t+u)-Y_{i}^{(\varepsilon)}(t)\right)\right\}$ exist for any $\beta \in \mathbb{R}$ and have an explicit form, namely, for $0 \leq t \leq t+u \leq T, i=1,2$,

$$
\begin{aligned}
\mathrm{E} \exp & \left\{\beta\left(Y_{i}^{(\varepsilon)}(t+u)-Y_{i}^{(\varepsilon)}(t)\right)\right\} \\
= & \begin{cases}\left(e^{\beta u_{i}^{(\varepsilon)}} p_{i}^{(\varepsilon)}+e^{-\beta u_{i}^{(\varepsilon)}} q_{i}^{(\varepsilon)}\right)^{[(t+u) / \varepsilon]-[t / \varepsilon]}, & \text { if } \varepsilon>0, \\
\exp \left\{\beta \mu_{i} u+\frac{\beta^{2} \sigma_{i}^{2} u}{2}\right\}, & \text { if } \varepsilon=0,\end{cases}
\end{aligned}
$$

where $p_{1}^{(\varepsilon)}=p_{++}^{(\varepsilon)}+p_{+-}^{(\varepsilon)}, q_{1}^{(\varepsilon)}=p_{-+}^{(\varepsilon)}+p_{--}^{(\varepsilon)}$ and $p_{2}^{(\varepsilon)}=p_{++}^{(\varepsilon)}+p_{-+}^{(\varepsilon)}, q_{2}^{(\varepsilon)}=p_{+-}^{(\varepsilon)}+p_{--}^{(\varepsilon)}$.

This makes it easy to check that condition $\mathbf{C}_{\mathbf{3}}$ holds for processes $\vec{Y}^{(\varepsilon)}(t)$ for any $\beta^{\prime}>\beta$.

Summarizing the remarks above, one can conclude that the conditions and, therefore, the statement of Theorem 4 hold, for the bivariate exponential price processes with independent increments $\vec{S}^{(\varepsilon)}(t)=\exp \left\{\vec{Y}^{(\varepsilon)}(t)\right\}, t \in[0, T]$ if condition $\mathbf{A}_{\mathbf{1}}$ holds for the corresponding payoff functions, i.e.,

$$
\Phi\left(\mathcal{M}_{\max , T}^{(\varepsilon)}\right) \rightarrow \Phi\left(\mathcal{M}_{\max , T}^{(0)}\right) \quad \text { as } \varepsilon \rightarrow 0 .
$$

Let assume for simplicity that $\varepsilon=T / N$ and consider the partition

$$
\Pi_{\varepsilon}=\left\{t_{0}=0<t_{1}=\varepsilon<\cdots<t_{N-1}=(N-1) \varepsilon<t_{N}=T\right\}
$$

on the interval $[0, T]$. In this case the Markov chain $\left(n, \vec{Y}^{(\varepsilon)}(n \varepsilon)\right), n=0,1, \ldots$, is a bivariate binomial tree model with the initial node $\left(0, \vec{y}_{0}\right)$ and nodes of the form $\left(n, \vec{y}_{n, l_{1}, l_{2}}\right)$, $l_{1}, l_{2}=0,1, \ldots, n$, after $n \geq 1$ steps. In the case of a non-reduced model with parameters of the approximating bivariate Bernoulli random vectors $\vec{Y}_{n}^{(\varepsilon)}$ defined by relations (7) and (9), the vector points $\vec{y}_{n, l_{1}, l_{2}}, l_{1}, l_{2}=0,1, \ldots, n, n=0,1, \ldots, N$, should be defined by the formula

$$
\vec{y}_{n, l_{1}, l_{2}}=\vec{y}_{0}+\left(\left(2 l_{1}-n\right) \sqrt{\varepsilon} \sqrt{\sigma_{1}^{2}+\mu_{1}^{2} \varepsilon},\left(2 l_{2}-n\right) \sqrt{\varepsilon} \sqrt{\sigma_{2}^{2}+\mu_{2}^{2} \varepsilon}\right) .
$$

In the case of a reduced model with parameters of the approximating bivariate Bernoulli random vectors $\left(Y_{n, 1}^{(\varepsilon)}, Y_{n, 2}^{(\varepsilon)}\right)$ defined by relations (7) and (12), the vector points $\vec{y}_{n, l_{1}, l_{2}}, l_{1}, l_{2}=0,1, \ldots, n, n=0,1, \ldots, N$, should be defined by the simpler formula,

$$
\vec{y}_{n, l_{1}, l_{2}}=\left(\left(2 l_{1}-n\right) \sqrt{\varepsilon} \sigma_{1},\left(2 l_{2}-n\right) \sqrt{\varepsilon} \sigma_{2}\right) .
$$

The corresponding tree has $(n+1)^{2}$ nodes appearing in the tree after the $n$-th step.

The standard backward procedure can be applied in order to find the optimal expected reward at moment 0 for the discrete time exponential bivariate binomial price process $\vec{S}^{(\varepsilon)}\left(t_{n}\right)=e^{\vec{Y}^{(\varepsilon)}\left(t_{n}\right)}, t_{n}=n \varepsilon, n=0,1, \ldots, N$. This optimal expected reward coincides, in this case, with the reward functional $\Phi\left(\mathcal{M}_{\Pi_{\varepsilon}, T}^{(\varepsilon)}\right)$ for the continuous time bivariate exponential price processes $\vec{S}^{(\varepsilon)}(t)=e^{\vec{Y}^{(\varepsilon)}(t)}, t \in[0, T]$. 


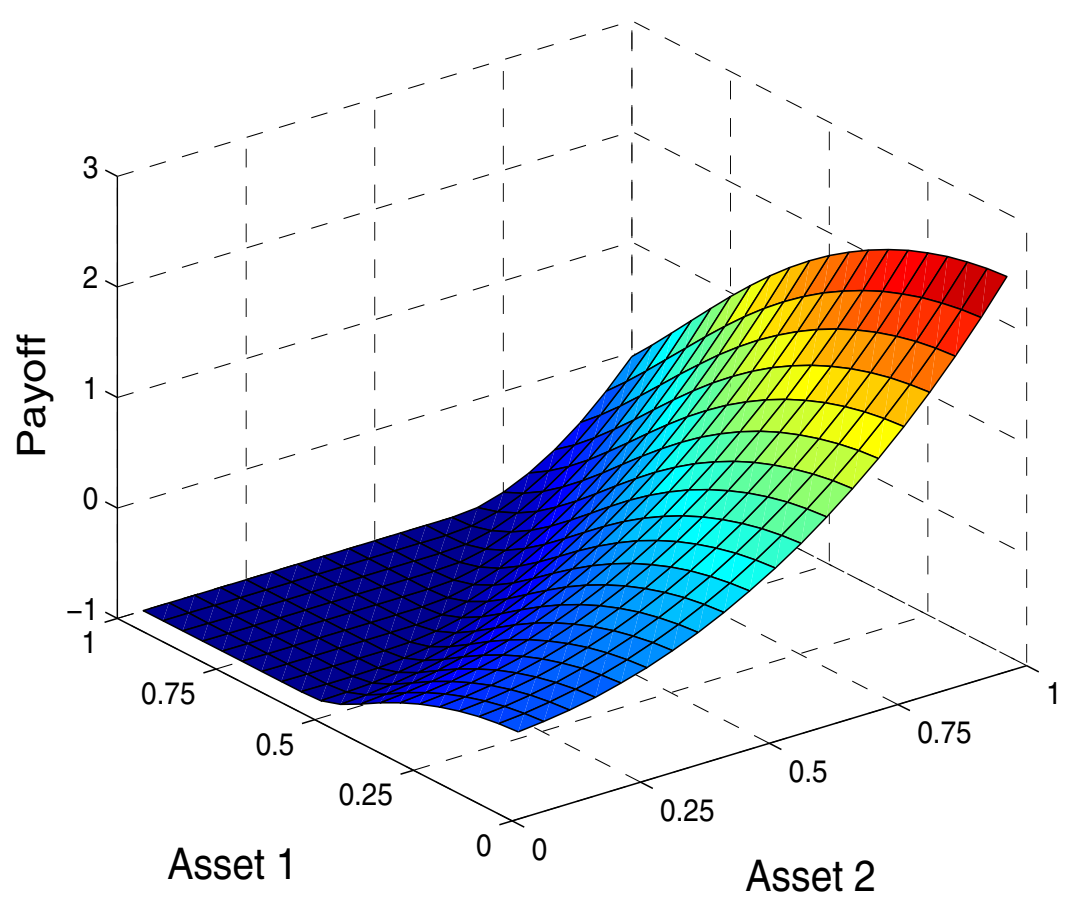

Figure 1. Rewards for exchange of Asset 1 for Asset 2, with volatilities $0.05 \leq \sigma_{1} \leq 1$ and $0.05 \leq \sigma_{2} \leq 1$.

To estimate the difference $\Phi\left(\mathcal{M}_{\max , T}^{(\varepsilon)}\right)-\Phi\left(\mathcal{M}_{\Pi_{\varepsilon}, T}^{(\varepsilon)}\right)$ we can use Theorem 3. In this case, $d\left(\Pi_{\varepsilon}\right)=\varepsilon$ and, for $\beta \in \mathbb{R}$ and $i=1,2$,

$$
\Delta_{\beta}\left(Y_{i}^{(\varepsilon)}(\cdot), \varepsilon, T\right)=\mathrm{E} \exp \left\{\beta\left|Y_{1, i}^{(\varepsilon)}\right|\right\}-1 \leq e^{\beta u_{i}^{(\varepsilon)}}-1
$$

Theorem 3 yields in this case the following relation:

$$
\begin{aligned}
& \Phi\left(\mathcal{M}_{\max , T}^{(\varepsilon)}\right)-\Phi\left(\mathcal{M}_{\Pi_{\varepsilon}, T}^{(\varepsilon)}\right) \\
& \quad \leq L_{3} \varepsilon+L_{4}\left(\left(e^{\beta u_{1}^{(\varepsilon)}}-1\right)^{\frac{\beta-\gamma}{\beta}}+\left(e^{\beta u_{2}^{(\varepsilon)}}-1\right)^{\frac{\beta-\gamma}{\beta}}\right) \rightarrow 0 \quad \text { as } \varepsilon \rightarrow 0 .
\end{aligned}
$$

As was pointed out in Section 3, the reward functional $\Phi\left(\mathcal{M}_{\Pi_{\varepsilon}, T}^{(\varepsilon)}\right)$ is the option optimal expected reward for American type options in discrete time that correspond to the discrete time Markov log-price process $\vec{Y}^{(\varepsilon)}\left(t_{n}\right), t_{n}=n \varepsilon, n=0,1, \ldots, N$, with parameter $\varepsilon=T / N$. Introduce the corresponding reward functions,

$$
w^{(\varepsilon)}\left(t_{n}, \vec{y}_{n, l_{1}, l_{2}}\right)=\sup _{t_{n} \leq \tau^{(\varepsilon)} \leq t_{N}=T} \mathrm{E}_{t_{n}, \vec{y}_{n, l_{1}, l_{2}}} g\left(\tau^{(\varepsilon)}, e^{\vec{Y}^{(\varepsilon)}\left(\tau^{(\varepsilon)}\right)}\right)
$$

where $\tau^{(\varepsilon)}$ is a discrete time Markov stopping moment such that event $\left\{\tau^{(\varepsilon)} \leq t_{k}\right\}$ depends only on trajectory $\vec{Y}^{(\varepsilon)}\left(t_{r}\right), t_{n} \leq t_{r} \leq t_{k}$ for $t_{n} \leq t_{k} \leq t_{N}$, and vector points $\vec{y}_{n, l_{1}, l_{2}}, l_{1}, l_{2}=0, \ldots, n, n=0, \ldots, N$, were defined in (15) , and, in particular, $\vec{y}_{0,0,0}=$ 
$\vec{y}_{0}=\left(y_{1}^{(0)}, y_{2}^{(0)}\right)$. Then,

$$
\Phi\left(\mathcal{M}_{\Pi_{\varepsilon}, T}^{(\varepsilon)}\right)=w^{(\varepsilon)}\left(0, \vec{y}_{0}\right) .
$$

The reward functions $w^{(\varepsilon)}\left(t_{n}, \vec{y}_{n, l_{1}, l_{2}}\right)$ can be found using the following backward recurrence relations:

$$
\left\{\begin{array}{r}
w^{(\varepsilon)}\left(t_{N}, \vec{y}_{N, l_{1}, l_{2}}\right)=g\left(t_{N}, e^{\left.\vec{y}_{N, l_{1}, l_{2}}\right)}, l_{1}, l_{2}=0, \ldots, N,\right. \\
w^{(\varepsilon)}\left(t_{n}, \vec{y}_{n, l_{1}, l_{2}}\right)=g\left(t_{n}, e^{\left.\vec{y}_{n, l_{1}, l_{2}}\right)}\right. \\
\vee\left(w^{(\varepsilon)}\left(t_{n+1}, \vec{y}_{n+1, l_{1}+1, l_{2}+1}\right) p_{++}^{(\varepsilon)}\right. \\
+w^{(\varepsilon)}\left(t_{n+1}, \vec{y}_{n+1, l_{1}+1, l_{2}}\right) p_{+-}^{(\varepsilon)} \\
+w^{(\varepsilon)}\left(t_{n+1}, \vec{y}_{n+1, l_{1}, l_{2}+1}\right) p_{-+}^{(\varepsilon)} \\
\left.+w^{(\varepsilon)}\left(t_{n+1}, \vec{y}_{n+1, l_{1}, l_{2}}\right) p_{--}^{(\varepsilon)}\right), \\
l_{1}, l_{2}=0, \ldots, n, \quad n=N-1, \ldots, 0 .
\end{array}\right.
$$

The corresponding approximation result for the bivariate binomial tree algorithm described above can be summarized in the following theorem.

Theorem 5. Let condition $\mathbf{A}_{1}$ hold and $|\rho|<1$. Then

$$
w^{(\varepsilon)}\left(0, \vec{y}_{0}\right)=\Phi\left(\mathcal{M}_{\Pi_{\varepsilon}, T}^{(\varepsilon)}\right) \rightarrow \Phi\left(\mathcal{M}_{\max , T}^{(0)}\right) \quad \text { as } \varepsilon \rightarrow 0 .
$$

Note that the asymptotic relation (21) takes place for any initial state $\vec{y}_{0} \in \mathbb{R}^{2}$.

Note that the modification of the approximation algorithm based on the reduced model described in (12) can be built and utilized in a similar way without assumption $|\rho|<1$.

Another example of a typical payoff function is a linear combination of payoff functions for a portfolio of options $g(t, \vec{s})=e^{-r t}\left(a_{1}\left[s_{1}-K_{1}\right]^{+}+a_{2}\left[s_{2}-K_{2}\right]^{+}\right)$.

Let us also mention the model of exchange of assets with payoff function $g(t, \vec{s})=$ $e^{-r t}\left(s_{1}-s_{2}\right)$. Note that this is an example of a payoff function which is not non-negative. The optimal stopping strategies for this model were recently studied in Mishura and Shevchenko 23 .

In both cases, the payoff functions are continuous, do not depend on the perturbation parameter $\varepsilon$, and obviously have a polynomial rate of growth. Thus, condition $\mathbf{A}_{1}$ automatically holds. Therefore, according to Theorem 5 the optimal expected reward functions for the above-described bivariate binomial exponential model converge to the corresponding optimal expected reward functionals for the corresponding bivariate geometric Brownian motion. It is useful to note that the results concerning bivariate binomial models admit an obvious generalization to the case of multivariate binomial models.

Let us give a numerical example for the model of exchange of assets with payoff function $g(t, \vec{s})=e^{-r t}\left(s_{1}-s_{2}\right)$. We consider the case when the holder of the option has the right to change Asset 1 for Asset 2. The option has maturity in 6 months. Asset 1 has an initial price 10 with a drift estimated to be 0.02 and volatility estimated to 0.1 per year. Asset 2 has an initial price 9.5 with a drift estimated to be 0.08 and volatility estimated to 0.35 per year. The correlation between the two assets are assumed to be $\rho=0.3$. The risk free interest rate is assumed to be $r=0.04$ for the time period of the contract.

Our studies show that tree size $N=100$ is enough, and the expected reward for a tree with size $N=100$ is 0.0850 . This should be compared with the expected reward for a tree with size $N=150$, which is 0.0858 . The calculation time for this tree size is 5.11 seconds 
on a $1.73 \mathrm{GHz}$ Intel ${ }^{\circledR}$ Pentium-M processor, $1 \mathrm{~GB}$ internal memory using Matlab ${ }^{\circledR}$. Figure 1 illustrates the reward for exchanging Asset 1 for Asset 2 when parameters of the model are as above, except the volatility varies on the interval $[0.05,1]$ for the two different assets. It is worth noting that for some combinations of volatility the reward will be negative, and thus exchange is not profitable. This question does, however, require an additional investigation.

\section{Binomial tree approximations for mean Reverse price processes}

Let us consider the model of price process introduced by Schwartz [26] for modeling energy prices. It has the following form:

$$
d \ln S(t)=-\alpha(\ln S(t)-\ln S(0)) d t+\nu d W(t), \quad t \geq 0,
$$

where $\alpha, \nu>0, W(t)$ is a standard Brownian motion, and the initial state $S(0)=s_{0}>0$ is a constant. We consider the case further when we want to price an American call option, that is, where a payoff function of the form $g(t, s)=e^{-r t}[s-K]^{+}$, where $r, K>0$. It is easy to check that the payoff function satisfies condition $\mathbf{A}_{\mathbf{1}}$ with some constants $K_{i}$, $i=1, \ldots, 5$, and parameters $\gamma_{0}=1$ and $\gamma_{1}=0$. Our object of interest in this case is the reward functional

$$
\Phi\left(\mathcal{M}_{\max , T}^{(0)}\right)=\sup _{0 \leq \tau \leq T} \mathrm{E} e^{-r \tau}[S(\tau)-K]^{+} .
$$

Equation (22) has the solution $S(t)=s_{0} \exp \left\{\nu e^{-\alpha t} \int_{0}^{t} e^{\alpha s} d W(s)\right\}, t \geq 0$. The process $S(t)$ is a diffusion process, which, however, can be represented as a non-random transformation of a simpler exponential Gaussian process with independent increments $S^{(0)}(t)=\exp \left\{\nu e^{-\alpha T} \int_{0}^{t} e^{\alpha s} d W(s)\right\}, t \geq 0$. It follows from the above formulas that $S(t)=s_{0}\left(S^{(0)}(t)\right)^{\exp \{\alpha(T-t)\}}, t \geq 0$. The processes $S(t), t \geq 0$, and $S^{(0)}(t), t \geq 0$, have the same natural filtration $\mathcal{F}_{t}, t \geq 0$, and therefore the class $\Phi\left(\mathcal{M}_{\max , T}^{(0)}\right)$ of all Markov moments $0 \leq \tau \leq T$ is also the same for these processes.

Let us now consider the transformed payoff function

$$
\tilde{g}^{(\varepsilon)}(t, s)=e^{-r t}\left[s_{0} s^{\exp \{\alpha(T-t)\}}-K\right]^{+} .
$$

This function also satisfies condition $\mathbf{A}_{\mathbf{1}}$ with some constants $K_{i}^{\prime}, i=1, \ldots, 5$, and parameters $\gamma_{0}=e^{\alpha T}+1, \gamma_{1}=e^{\alpha T}-1$. It follows from the remarks above that the reward functional

$$
\begin{aligned}
\Phi\left(\mathcal{M}_{\max , T}^{(0)}\right) & =\sup _{0 \leq \tau \leq T} \mathrm{E} e^{-r \tau}[S(\tau)-K]^{+} \\
& =\sup _{0 \leq \tau \leq T} \mathrm{E} e^{-r \tau}\left[s_{0} S^{(0)}(\tau)^{\exp \{\alpha(T-\tau)\}}-K\right]^{+} .
\end{aligned}
$$

The process $S^{(0)}(t)=e^{Y^{(0)}(t)}, t \geq 0$, where $Y^{(0)}(t)=\nu e^{-\alpha T} \int_{0}^{t} e^{\alpha s} d W(s), t \geq 0$, is a Gaussian process with independent increments.

We approximate the process $Y^{(0)}(t), t \geq 0$, with a trinomial sum-process $Y^{(\varepsilon)}(t)=$ $\sum_{1 \leq k \leq[t / \varepsilon]} Y_{n}^{(\varepsilon)}, t \geq 0$, where $Y_{n}^{(\varepsilon)}, n=1,2, \ldots$, are, for every $\varepsilon>0$, independent random variables that have the following structure:

$$
Y_{n}^{(\varepsilon)}=\left\{\begin{array}{ccc}
u^{(\varepsilon)} & & p_{n,+}^{(\varepsilon)} \\
0 & \text { with prob. } & p_{n, \cdot}^{(\varepsilon)} \\
-u^{(\varepsilon)} & p_{n,-}^{(\varepsilon)}
\end{array}\right.
$$

Let us assume that $\varepsilon=T / N$. 
In order to fit the trinomial sum-processes $Y^{(\varepsilon)}(t)$ to the limit process $Y^{(0)}(t)$, we should fit expectation and variance for the random variables $Y_{n}^{(\varepsilon)}$ and increment of the limit process $Y^{(0)}(n \varepsilon)-Y^{(0)}((n-1) \varepsilon)$ for $n=1, \ldots, N$.

Simple calculations show that

$$
\mathrm{E}\left(Y^{(0)}(n \varepsilon)-Y^{(0)}((n-1) \varepsilon)\right)=\mathrm{E} \nu e^{-\alpha T} \int_{(n-1) \varepsilon}^{n \varepsilon} \nu e^{\alpha s} d W(s)=0
$$

and

$$
\begin{aligned}
\sigma_{n, \varepsilon}^{2} & =\operatorname{Var}\left(Y^{(0)}(n \varepsilon)-Y^{(0)}((n-1) \varepsilon)\right) \\
& =\operatorname{Var} \nu e^{-\alpha T} \int_{(n-1) \varepsilon}^{n \varepsilon} e^{\alpha s} d W(s)=\nu^{2} e^{-2 \alpha T} e^{2 \alpha n \varepsilon} \frac{1-e^{-2 \alpha \varepsilon}}{2 \alpha} .
\end{aligned}
$$

The following system of $3 N$ equations and $3 N+1$ unknowns should be solved:

$$
\left\{\begin{array}{l}
\mathrm{E} Y_{n}^{(\varepsilon)}=u^{(\varepsilon)}\left(p_{n,+}^{(\varepsilon)}-p_{n,-}^{(\varepsilon)}\right)=0, \\
\operatorname{Var} Y_{n}^{(\varepsilon)}=\left(u^{(\varepsilon)}\right)^{2}\left(p_{n,+}^{(\varepsilon)}+p_{n,-}^{(\varepsilon)}\right)=\sigma_{n, \varepsilon}^{2}, \\
p_{n,+}^{(\varepsilon)}+p_{n,-}^{(\varepsilon)}+p_{n, \cdot}^{(\varepsilon)}=1, \\
n=1, \ldots, N
\end{array}\right.
$$

The system above has a solution of the following form:

$$
\left\{\begin{array}{l}
u^{(\varepsilon)}=\kappa \sqrt{\varepsilon} \\
p_{n,+}^{(\varepsilon)}=p_{n,-}^{(\varepsilon)}=\frac{\sigma_{n, \varepsilon}^{2}}{2 \kappa^{2} \varepsilon} \\
p_{n, \cdot}^{(\varepsilon)}=1-\frac{\sigma_{n, \varepsilon}^{2}}{\kappa^{2} \varepsilon} \\
n=1, \ldots, N
\end{array}\right.
$$

Let us consider the probability $p_{n,+}^{(\varepsilon)}=\sigma_{n, \varepsilon}^{2} /\left(2 \kappa^{2} \varepsilon\right)$. It is easy to show that

$$
\nu^{2} e^{-2 \alpha T} \leq \frac{\sigma_{n, \varepsilon}^{2}}{\varepsilon} \leq \nu^{2} e^{-2 \alpha T} e^{2 \alpha T}=\nu^{2}
$$

for $n=1, \ldots, N$. Thus, it is possible to choose $\kappa$ large enough such that the values of probabilities

$$
0 \leq \frac{\nu^{2} e^{-2 \alpha T}}{2 u^{2}} \leq p_{n,+}^{(\varepsilon)} \leq \frac{\nu^{2}}{2 u^{2}} \leq \frac{1}{2}
$$

for $n=1, \ldots, N$. In fact one can take any $\kappa \geq \nu$.

The defining relation (25) implies that for any $\delta>0$ if $\varepsilon$ is small enough, namely, if $\kappa \sqrt{\varepsilon} \leq \delta$, then

$$
\sum_{n \leq[T / \varepsilon]} \mathrm{P}\left\{\left|Y_{n}^{(\varepsilon)}\right|>\delta\right\}=0 .
$$

Also, by the definition of processes $Y^{(\varepsilon)}(t)$, for $\varepsilon \geq 0$ and $0 \leq t \leq T$,

$$
\mathrm{E} Y^{(\varepsilon)}(t)=0,
$$

and, for every $0 \leq t \leq T$,

$$
\begin{aligned}
\operatorname{Var} Y^{(\varepsilon)}(t) & =\nu^{2} e^{-2 \alpha T} \int_{0}^{[t / \varepsilon] \varepsilon} e^{2 \alpha s} d s=\nu^{2} e^{-2 \alpha T} \frac{e^{2 \alpha[t / \varepsilon] \varepsilon}-1}{2 \alpha} \\
& \rightarrow \operatorname{Var} Y^{(0)}(t)=\nu^{2} e^{-2 \alpha T} \frac{e^{2 \alpha t}-1}{2 \alpha} \text { as } 0<\varepsilon \rightarrow 0 .
\end{aligned}
$$

Since the functions in (32) are monotone and the corresponding limit function is continuous, this convergence is also uniform in the interval $[0, T]$ and, therefore, conditions of 
the Ascoli-Arzelá theorem, in particular, conditions of compactness in uniform topology, hold as $\varepsilon \rightarrow 0$.

The remarks above imply that conditions of convergence theorems for vector sumprocesses with independent increments given, for example, in Skorokhod [33] hold for processes $\vec{Y}^{(\varepsilon)}(t), t \in[0, T]$. Thus, the processes $Y^{(\varepsilon)}(t), t \in[0, T]$, weakly and, moreover, J-converge to the process $Y^{(0)}(t), t \in[0, T]$, as $\varepsilon \rightarrow 0$.

Therefore, conditions $\mathbf{B}_{\mathbf{2}}$ and $\mathbf{D}_{\mathbf{2}}$ hold for step-sum processes with independent increments $Y^{(\varepsilon)}(t)$.

Also, the moment generation function $\operatorname{E} \exp \left\{\beta\left(Y^{(\varepsilon)}(t+s)-Y^{(\varepsilon)}(t)\right)\right\}$ exists for any $\beta \in \mathbb{R}$ and has an explicit form, namely, for $0 \leq t \leq t+s \leq T$,

$$
\begin{aligned}
& \mathrm{E} \exp \left\{\beta\left(Y^{(\varepsilon)}(t+s)-Y^{(\varepsilon)}(t)\right)\right\} \\
& = \begin{cases}\prod_{[t / \varepsilon]+1}^{[(t+s) / \varepsilon]}\left(e^{\beta \kappa \sqrt{\varepsilon}} p_{n,+}^{(\varepsilon)}+e^{-\beta \kappa \sqrt{\varepsilon}} p_{n,-}^{(\varepsilon)}+p_{n, \cdot}^{(\varepsilon)}\right) & \text { if } \varepsilon>0, \\
\exp \left\{\frac{1}{2} \beta^{2} \nu^{2} e^{-2 \alpha T} \int_{t}^{t+s} e^{2 \alpha v} d v\right\} & \text { if } \varepsilon=0 .\end{cases}
\end{aligned}
$$

Using formula (33) it is possible to check that condition $\mathbf{C}_{\mathbf{3}}$ holds for any $\beta^{\prime}>\beta$. Indeed, since $\nu \leq \kappa$ and $\sigma_{n, \varepsilon}^{2} /\left(2 \kappa^{2} \varepsilon\right) \leq \frac{1}{2}$ for $n=1, \ldots, N$, we get

$$
\begin{aligned}
& \Xi_{ \pm \beta^{\prime}}^{\prime}\left(Y^{(\varepsilon)}(\cdot), T\right)=\sup _{0 \leq t \leq t+u \leq T} \mathrm{E} \exp \left\{ \pm \beta^{\prime}\left(Y^{(\varepsilon)}(t+u)-Y^{(\varepsilon)}(t)\right)\right\} \\
& \leq \prod_{n=1}^{[T / \varepsilon]}\left(1+\frac{\sigma_{n, \varepsilon}^{2}}{2 \kappa^{2} \varepsilon}\left(e^{\beta^{\prime} \kappa \sqrt{\varepsilon}}+e^{-\beta^{\prime} \kappa \sqrt{\varepsilon}}-2\right)\right) \\
& \leq\left(1+\frac{1}{2}\left(e^{\beta^{\prime} \kappa \sqrt{\varepsilon}}+e^{-\beta^{\prime} \kappa \sqrt{\varepsilon}}-2\right)\right)^{T / \varepsilon} \rightarrow \exp \left\{\frac{\beta^{\prime 2} \kappa^{2} T}{2}\right\}<\infty \quad \text { as } \varepsilon \rightarrow 0 .
\end{aligned}
$$

Therefore, all conditions of Theorem 4 hold for log-price processes $Y^{(\varepsilon)}(t)$ and, therefore,

$$
\Phi\left(\mathcal{M}_{\max , T}^{(\varepsilon)}\right) \rightarrow \Phi\left(\mathcal{M}_{\max , T}^{(0)}\right) \quad \text { as } \varepsilon \rightarrow 0 .
$$

Let us now consider the partition

$$
\Pi_{\varepsilon}=\left\{t_{0}=0<t_{1}=\varepsilon<\cdots<t_{N-1}=(N-1) \varepsilon<t_{N}=T\right\}
$$

on the interval $[0, T]$. In this case, the Markov chain $\left(n, Y^{(\varepsilon)}(n \varepsilon)\right), n=0,1, \ldots$, is a trinomial tree model with the initial node $(0,0)$ and nodes of the form

$$
\left(n, y_{n, l}\right), \quad l=0, \pm 1, \ldots, \pm n,
$$

after $n \geq 1$ steps. In the case of a model with parameters of the approximating trinomial random variables $Y_{n}^{(\varepsilon)}$ defined by relations (25) and (29), the points

$$
y_{n, l}, \quad l=0, \pm 1, \ldots, \pm n, n=0,1, \ldots, N,
$$

should be defined by the formula

$$
y_{n, l}=l \sqrt{\varepsilon} \kappa .
$$

The corresponding tree has $2 n+1$ nodes after $n$ steps. The number of nodes is a linear function of $n$.

The standard backward procedure can be applied in order to find the optimal expected reward at moment 0 for the discrete time exponential trinomial price process $S^{(\varepsilon)}\left(t_{n}\right)=$ $\exp \left\{Y^{(\varepsilon)}\left(t_{n}\right)\right\}, t_{n}=n \varepsilon, n=0,1, \ldots, N$. This optimal expected reward coincides, in this 
case, with the reward functional $\Phi\left(\mathcal{M}_{\Pi_{\varepsilon}, T}^{(\varepsilon)}\right)$ for the continuous time exponential price processes $S^{(\varepsilon)}(t)=\exp \left\{Y^{(\varepsilon)}(t)\right\}, t \in[0, T]$.

To estimate the difference $\Phi\left(\mathcal{M}_{\max , T}^{(\varepsilon)}\right)-\Phi\left(\mathcal{M}_{\Pi_{\varepsilon}, T}^{(\varepsilon)}\right)$ we can use Theorem 3. In this case, $d\left(\Pi_{\varepsilon}\right)=\varepsilon$, and for $\beta \in \mathbb{R}$,

$$
\Delta_{\beta}\left(Y^{(\varepsilon)}(\cdot), \varepsilon, T\right)=\max _{1 \leq n \leq N}\left(\mathrm{E} \exp \left\{\beta\left|Y_{n}^{(\varepsilon)}\right|\right\}-1\right) \leq e^{\beta \kappa \sqrt{\varepsilon}}-1 .
$$

In this case Theorem 3 yields the following relation:

$$
\Phi\left(\mathcal{M}_{\max , T}^{(\varepsilon)}\right)-\Phi\left(\mathcal{M}_{\Pi_{\varepsilon}, T}^{(\varepsilon)}\right) \leq L_{3} \varepsilon+L_{4}\left(e^{\beta \kappa \sqrt{\varepsilon}}-1\right)^{(\beta-\gamma) / \beta} \rightarrow 0 \quad \text { as } \varepsilon \rightarrow 0 .
$$

As was pointed out in Section 3, the reward functional $\Phi\left(\mathcal{M}_{\Pi_{\varepsilon}, T}^{(\varepsilon)}\right)$ is the option optimal expected reward for American type options in discrete time that correspond to the discrete time Markov log-price process $Y^{(\varepsilon)}\left(t_{n}\right), t_{n}=n \varepsilon, n=0,1, \ldots, N$, with parameter $\varepsilon=T / N$. Introduce the corresponding reward functions,

$$
\begin{aligned}
& w^{(\varepsilon)}\left(t_{n}, y_{n, l}\right) \\
& \quad=\sup _{t_{n} \leq \tau^{(\varepsilon)} \leq t_{N}=T} \mathrm{E}_{t_{n}, y_{n, l}} e^{-r \tau^{(\varepsilon)}}\left[s_{0} \exp \left\{Y^{(\varepsilon)}\left(\tau^{(\varepsilon)}\right) \exp \left\{\alpha\left(T-\tau^{(\varepsilon)}\right)\right\}\right\}-K\right]^{+},
\end{aligned}
$$

where $\tau^{(\varepsilon)}$ is a discrete time Markov stopping moment such that event $\left\{\tau^{(\varepsilon)} \leq t_{k}\right\}$ depends only on trajectory $\vec{Y}^{(\varepsilon)}\left(t_{r}\right), t_{n} \leq t_{r} \leq t_{k}$, for $t_{n} \leq t_{k} \leq t_{N}$, and $y_{l}=l \sqrt{\varepsilon} u$, $l=0, \pm 1, \ldots, \pm n, n=0, \ldots, N$. Then,

$$
\Phi\left(\mathcal{M}_{\Pi_{\varepsilon}, T}^{(\varepsilon)}\right)=w^{(\varepsilon)}(0,0) .
$$

The reward functions $w^{(\varepsilon)}\left(t_{n}, y_{l}\right)$ can be found using the following backward recurrence relations:

$$
\left\{\begin{aligned}
w^{(\varepsilon)}\left(t_{N}, y_{N, l}\right)= & e^{-r t_{N}}\left[s_{0} e^{y_{N, l}}-K\right]^{+}, l=0, \pm 1, \ldots, \pm N \\
w^{(\varepsilon)}\left(t_{n}, y_{n, l}\right)= & e^{-r t_{n}}\left[s_{0} \exp \left\{y_{n, l} e^{\alpha\left(T-t_{n}\right)}\right\}-K\right]^{+} \\
& \vee\left(w^{(\varepsilon)}\left(t_{n+1}, y_{n+1, l+1}\right) p_{n,+}^{(\varepsilon)}+w^{(\varepsilon)}\left(t_{n+1}, y_{n+1, l}\right) p_{n, \cdot}^{(\varepsilon)}\right. \\
& \left.+w^{(\varepsilon)}\left(t_{n+1}, y_{n+1, l-1}\right) p_{n,-}^{(\varepsilon)}\right) \\
& l=0, \pm 1, \ldots, \pm n, n=N-1, \ldots, 0 .
\end{aligned}\right.
$$

The corresponding convergence result of the Schwartz model can be summarized in the following theorem.

Theorem 6. Let the price process correspond to the Schwartz model and consider an American option that has the standard payoff function. Then

$$
w^{(\varepsilon)}(0,0)=\Phi\left(\mathcal{M}_{\Pi_{\varepsilon}, T}^{(\varepsilon)}\right) \rightarrow \Phi\left(\mathcal{M}_{\max , T}^{(0)}\right) \quad \text { as } \varepsilon \rightarrow 0 .
$$

Note that the asymptotic relation (41) takes place for any initial state $s_{0} \in \mathbb{R}_{+}^{1}$.

Note also that in the above described approximation tree algorithm as well as in Theorem 6 , the standard payoff function $g(t, s)=e^{-r t}[s-K]^{+}$can be replaced by any payoff function satisfying condition $\mathbf{A}_{\mathbf{1}}$.

Let us now consider a numerical example when a standard American call option written on a commodity is assumed to follow the Schwartz model. The commodity is currently traded at 10 and has an estimated volatility of 0.25 and mean reverting coefficient of $\alpha=1$. The option has a strike price $K=11$ and maturity $T=0.5$ years. Finally, the risk free interest rate is $r=0.04$. 


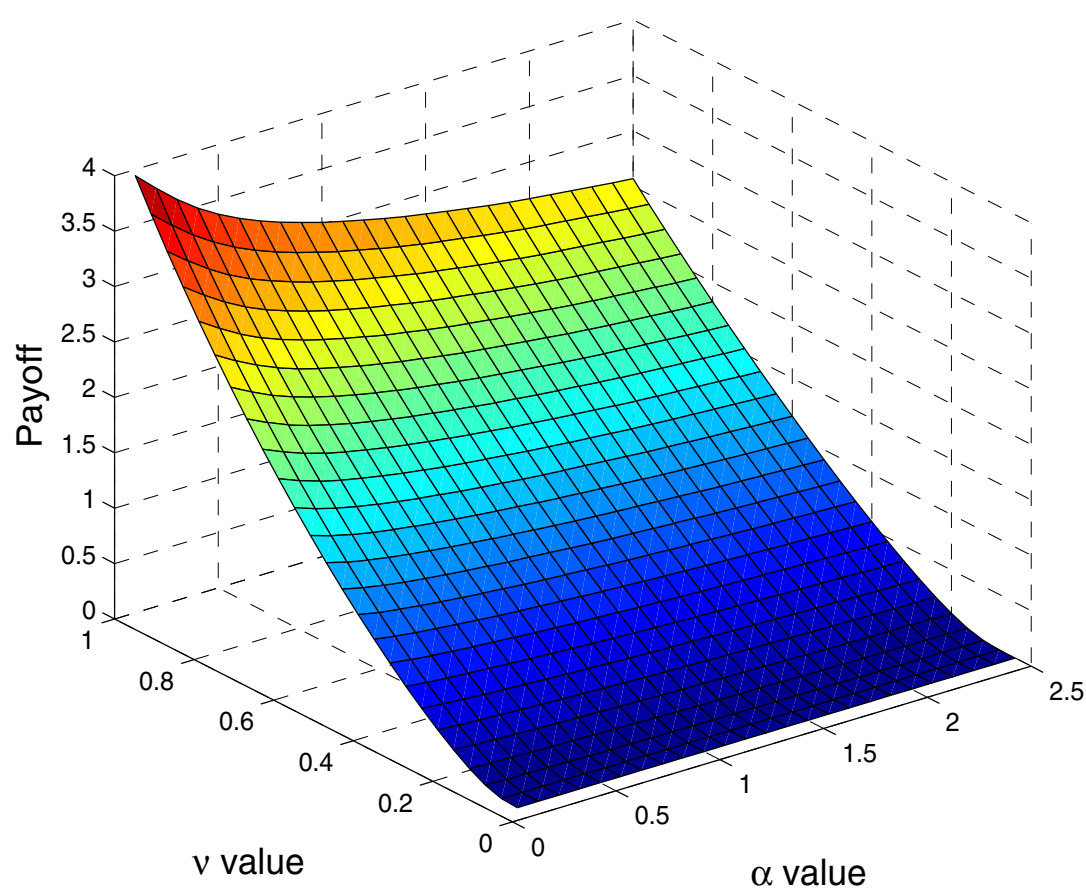

FiguRE 2. Reward of an American option on a commodity assumed to follow the Schwartz model and having parameters $0.1 \leq \alpha \leq 2.5$ and $0.04 \leq \nu \leq 1$

Our studies show that tree size $N=50$ is enough and that the expected reward for a tree with size $N=50$ is 0.2802 . This should be compared with the expected reward for a tree with size $N=100$ which is 0.2863 . The calculation time for this tree size is 0.963 seconds on a $1.73 \mathrm{GHz}$ Intel ${ }^{\circledR}$ Pentium-M processor, 1GB internal memory using Matlab ${ }^{\circledR}$.

For low values of the $\nu$ parameter the option reward reaches its minimum, and when the $\nu$ value are high and the $\alpha$ value are low, the option reward reaches its maximum.

\section{BIBLIOGRAPHY}

1. K. Amin and A. Khanna, Convergence of American option values from discrete- to continuoustime financial models, Math. Finance 4 (1994), no. 4, 289-304. MR1299240 (95h:90017)

2. F. Coquet and S. Toldo, Convergence of values in optimal stopping and convergence in optimal stopping times, Electr. J. Probab. 12, no. 8 (2007), 207-228. MR.2299917 (2007k:60120)

3. G. Cortazar, M. Gravet, and J. Urzua, The valuation of multidimensional American real options using the LSM simulation method, Comp. Oper. Res. 35 (2008), 113-129.

4. S. Dayanik and I. Karatzas, On the optimal stopping problem for one-dimensional diffusions, Stoch. Process. Appl. 107 (2003), 173-212. MR1999788 (2004d:60104)

5. P. Dupuis and $\mathrm{H}$. Wang, On the convergence from discrete to continuous time in an optimal stopping problem, Ann. Appl. Probab. 15 (2005), 1339-1366. MR2134106 (2006b:60081)

6. E. Ekström, C. Lindberg, J. Tysk, and H. Wanntorp, Optimal Liquidation of a Call Spread, Preprint, Uppsala University, Sweden, 2009. MR2668508 (2011i:91159)

7. B. Gau, J. Z. Huang, and M. Subrahmanyam, The valuation of American barrier options using the decomposition technique, Econom. Dynam. Control 24 (2000), 1783-1827. MR1784574 (2001e:91083) 
8. V. Henderson and D. Hobson, An explicit solution for an optimal stopping/optimal control problem which models an asset sale, Ann. Appl. Probab. 18 (2008), 1681-1705. MR2462545 (2010b:60122)

9. S. D. Jacka, Optimal stopping and the American put, Math. Finance 1 (1991), 1-14.

10. H. Jönsson, Monte Carlo studies of American type options with discrete time, Theory Stoch. Process. 7(23) (2001), no. 1-2, 163-188.

11. H. Jönsson, Optimal Stopping Domains and Reward Functions for Discrete Time American Type Options, Ph.D. Thesis, vol. 22, Mälardalen University, 2005.

12. H. Jönsson, A. G. Kukush, and D. S. Silvestrov, Threshold structure of optimal stopping strategies for American type options. I, Theor. Ǐmovirn. Mat. Stat. 71 (2004), 113-123; English transl. in Theory Probab. Math. Statist. 71 (2005), 93-103. MR2144323 (2006h:91075)

13. H. Jönsson, A. G. Kukush, and D. S. Silvestrov, Threshold structure of optimal stopping strategies for American type options. II, Theor. Ǐmovirn. Mat. Stat. 72 (2005), 42-53; English transl. in Theory Probab. Math. Statist. 72 (2006) 47-58. MR2168135 (2006i:62070)

14. I. Kim, The analytic valuation of American options, Rev. Financ. Studies 3 (1990), 547-572.

15. A. G. Kukush and D. S. Silvestrov, Structure of optimal stopping strategies for American type options, Probabilistic Constrained Optimization: Methodology and Applications (S. Uryasev, ed.), Kluwer, Dordrecht, 2000, pp. 173-185. MR1819411

16. A. G. Kukush and D. S. Silvestrov, Skeleton approximation of optimal stopping strategies for American type options with continuous time, Theory Stoch. Process. 7(23) (2001), no. 1-2, $215-230$.

17. A. G. Kukush and D. S. Silvestrov, Optimal price of American type options with discrete time, Theory Stoch. Process. 10(26) (2004), no. 1-2, 72-96. MR.2327852

18. R. Lundgren, Structure of optimal stopping domains for American options with knock out domains, Theory Stoch. Process. 13(29) (2007), no. 4 , 98-129. MR2482254(2009m:91088)

19. R. Lundgren, Convergence of Option Rewards, Ph.D. Thesis, vol. 89, Mälardalen University, 2010.

20. R. Lundgren and D. S. Silvestrov, Convergence of Option Rewards for Multivariate Price Processes, Research Report 2009:10, Department of Mathematics, Stockholm University.

21. R. Lundgren and D. Silvestrov, Optimal stopping and reselling of European options, Mathematical and Statistical Models and Methods in Reliability (V. Rykov, N. Balakrishan, and M. Nikulin, eds.), Birkhäuser, Boston, 2010, 371-390. MR2797677 (2012d:60127)

22. R. Lundgren, D. S. Silvestrov, and A.G. Kukush, Reselling of options and convergence of option rewards, J. Numer. Appl. Math., 1(96), no. 3-4 (2008), 149-172.

23. Yu. Mishura and G. Shevchenko, The optimal time to exchange one asset for another on finite interval, Optimality and Risk-Modern Trends in Mathematical Finance (F. Delbaen, M. Rasonyi, and C. Stricker, eds.), Springer, 2009, pp. 197-210. MR2648604(2011h:60096)

24. G. Peskir and A. Shiryaev, Optimal Stopping and Free-Boundary Problems, Birkhäuser, Basel, 2006. MR2256030 (2008d:60004)

25. J. L. Prigent, Weak Convergence of Financial Markets, Springer, New York, 2003. MR2036683 (2005b:91007)

26. E. S. Schwartz, The stochastic behaviour of commodity prices: implications for valuation and hedgeing, J. Finance. 52 (1997), no. 3, 923-973.

27. D. Silvestrov, V. Galochkin, and A. Malyarenko, OPTAN-a pilot program system for analysis of options, Theory Stoch. Process. 7(23) (2001), no. 1-2, 291-300.

28. D. S. Silvestrov, V. G. Galochkin, and V. G. Sibirtsev, Algorithms and programs for optimal Monte Carlo pricing of American type options, Theory Stoch. Process. 5(21) (1999), no. 1-2, $175-187$.

29. D. S. Silvestrov, H. Jönsson, and F. Stenberg, Convergence of option rewards for Markov type price processes controlled by stochastic indices. 1, Research Report 2006-1, Department of Mathematics and Physics, Mälardalen University.

30. D. Silvestrov, H. Jönsson, and F. Stenberg, Convergence of option rewards for Markov type price processes, Theory Stoch. Process. 13(29) (2007), no. 4, 174-185. MR2482260 (2009m:60155)

31. D. S. Silvestrov, H. Jönsson, and F. Stenberg, Convergence of option rewards for Markov type price processes modulated by stochastic indices. I, Teor. İmovirn. Mat. Stat. 79 (2008), 149-165; English transl. in Theory Probab. Math. Statist. 79 (2009), 153-170. MR2494545(2010e:60151)

32. D. S. Silvestrov, H. Jönsson, F. Stenberg, Convergence of option rewards for Markov type price processes modulated by stochastic indices. II, Teor. İmovirn. Mat. Stat. 80 (2009), 138-155; English transl. in Theory Probab. Math. Statist. 80 (2010), 153-172. MR2541960(2010h:60207) 
33. A. V. Skorokhod, Random Processes with Independent Increments, Probability Theory and Mathematical Statistics, "Nauka", Moscow, 1964; English transl., Nat. Lending Library for Sci. and Tech., Boston Spa, 1971. MR860563 (88b:60171)

34. F. Stenberg, Semi-Markov Models for Insurance and Option Rewards, Ph.D. Thesis, vol. 38, Mälardalen University, 2006.

35. Z. Zhang and K-G. Lim, A non-lattice pricing model of American options under stochastic volatility, J. Futures Markets 26, (2006), no. 5, 417-448.

Stockholm University, Sweden

E-mail address: silvestrov@math.su.se

MÄlardalen UNIVERSity, VÄSTERÅs, SWEdEN

E-mail address: robin.lundgren@mdh.se

Received 04/APR/2011

Originally published in English 